\title{
ARTICLE OPEN Synchronous inhibition of mTOR and VEGF/NRP1 axis impedes tumor growth and metastasis in renal cancer
}

\author{
Krishnendu Pal ${ }^{1,2}$, Vijay Sagar Madamsetty iD $^{1,2}$, Shamit Kumar Dutta ${ }^{1}$, Enfeng Wang ${ }^{1}$, Ramcharan Singh Angom ${ }^{1}$ and \\ Debabrata Mukhopadhyay ${ }^{1 *}$
}

\begin{abstract}
Clear cell renal cell carcinoma (ccRCC) is known for its highly vascular phenotype which is associated with elevated expression of vascular endothelial growth factor A (VEGF), also known as vascular permeability factor (VPF). Accordingly, VEGF has been an attractive target for antiangiogenic therapies in ccRCC. Two major strategies have hitherto been utilized for VEGF-targeted antiangiogenic therapies: targeting VEGF by antibodies, ligand traps or aptamers, and targeting the VEGF receptor signaling via antibodies or small-molecule tyrosine-kinase inhibitors (TKIs). In the present article we utilized two entirely different approaches: targeting mammalian target of rapamycin (mTOR) pathway that is known to be involved in VEGF synthesis, and disruption of VEGF/ Neuroplin-1 (NRP1) axis that is known to activate proangiogenic and pro-tumorigenic signaling in endothelial and tumor cells, respectively. Everolimus (E) and a small-molecule inhibitor EG00229 (G) were used for the inhibition of mTOR and the disruption of VEGF/NRP1 axis, respectively. We also exploited a liposomal formulation decorated with a proprietary tumor-targeting-peptide (TTP) to simultaneously deliver these two agents in a tumor-targeted manner. The TTP-liposomes encapsulating both Everolimus and EG00229 (EG-L) demonstrated higher in vitro and in vivo growth retardation than the single drug-loaded liposomes (E-L and G$\mathrm{L}$ ) in two different $\mathrm{CCRCC}$ models and led to a noticeable reduction in lung metastasis in vivo. In addition, EG-L displayed remarkable inhibition of tumor growth in a highly aggressive syngeneic immune-competent mouse model of ccRCC developed in Balb/c mice. Taken together, this study demonstrates an effective approach to achieve improved therapeutic outcome in ccRCC.
\end{abstract}

npj Precision Oncology (2019)3:31; https://doi.org/10.1038/s41698-019-0105-2

\section{INTRODUCTION}

Clear cell renal cell carcinoma (ccRCC) is the most prevailing (75-80\%) subtype of RCC, which, in turn, accounts for approximately $90 \%$ of all kidney cancers. ${ }^{1,2}$ The von Hippel-Lindau (VHL) tumor suppressor gene is often inactivated in cCRCC, leading to the stabilization and consequent accumulation of hypoxiainducible-factor-1a (HIF-1 a) ${ }^{3}$ and overexpression of VEGF (also known as VPF). ${ }^{4,5}$ This VHL gene inactivation and high VEGF expression in RCC have been correlated with tumor aggressiveness and poor survival. ${ }^{6}$ VEGF plays a critical role in both normal and tumor-associated angiogenesis via stimulation of endothelial cell proliferation and migration, ${ }^{7}$ protection of endothelial cells from apoptosis, ${ }^{8}$ and reversal of senescence in endothelial cells. ${ }^{9}$ VEGF is known to exert its effect through the interaction with transmembrane tyrosine-kinase receptors that include VEGF receptor 1 (VEGFR1), VEGF receptor 2 (VEGFR2), and Neuropilin-1 (NRP1). ${ }^{10}$ Naturally, all of these receptors are expressed in vascular endothelial cells. However, NRP1 is also expressed in other cells including tumor cells ${ }^{11}$ and immune cells. ${ }^{12}$ Although, VEGFR2 is well established as the key receptor behind the proangiogenic signaling of VEGF, ${ }^{13}$ NRP1 has been shown to play an essential role in VEGF induced endothelial cell migration. ${ }^{14}$ In addition, several recent studies implicated VEGF/NRP1 axis in tumor-cell autocrine signaling pathways responsible for imparting cancer stemness. ${ }^{15-20}$

With the advent of the concept of antiangiogenic therapy, ${ }^{21}$ targeting VEGF became a lucrative option for the treatment of cancer, especially in highly angiogenic types such as ccRCC. Majority of hitherto used VEGF-targeted antiangiogenic therapies fall in two main categories. ${ }^{22}$ The first approach targets VEGF directly utilizing monoclonal antibodies, soluble receptor/ligand traps or aptamers, while the other strategy inhibits VEGF signaling by targeting VEGFR2 with monoclonal antibodies or smallmolecule tyrosine-kinase inhibitors (TKIs). Among the clinically approved VEGF-targeting agents, the humanized anti-VEGF monoclonal antibody Bevacizumab falls under the first category while the small-molecule VEGFR-TKIs such as Sorafenib and Sunitinib are examples of the latter. Initially, these treatment regimens provided a paradigm shift for the treatment of cCRCC. However, patients ultimately became resistant towards the antiangiogenic therapies, thus limiting the long-term benefits. ${ }^{23}$

In addition to the above anti-VEGF treatments, several other routes have been evaluated for antiangiogenic therapy. For instance, mTOR inhibitors have been shown to inhibit hypoxiaor growth factor-induced endothelial cell proliferation, migration, and tube formation in in vitro studies. ${ }^{24}$ Likewise, the antiangiogenic efficacy of mTOR inhibitors have been validated in a variety of cancer models in vivo. ${ }^{25-28}$ Inhibition of mTOR induces apoptosis in tumor-associated endothelial cells that ultimately leads to significant reductions in microvessel density and tumor growth. In addition, mTOR inhibitors can also impact angiogenesis by inhibiting the production of proangiogenic factors in tumors and tumor-associated stromal cells. ${ }^{29}$ Moreover, mTOR contributes in the hypoxic tumor response by stabilizing hypoxia-inducible factor-1a (HIF-1 a), ${ }^{30}$ hence it is not surprising that mTOR inhibitors can reduce VEGF expression and act as antiangiogenic agents. $^{31}$

Recently, several articles described the use of VEGF/NRP1 axis inhibitors, ranging from peptide fragments to small-molecule

${ }^{1}$ Department of Biochemistry and Molecular Biology, Mayo Clinic Florida, 4500 San Pablo Road S, Jacksonville, FL 32224, USA. ${ }^{2}$ These authors contributed equally: Krishnendu Pal, Vijay Sagar Madamsetty. *email: mukhopadhyay.debabrata@mayo.edu 
inhibitors, as an alternative therapeutic strategy for cancer. ${ }^{32-38}$ Among them, one small-molecule inhibitor, EG00229, demonstrated remarkable growth inhibition in glioma and lung cancer via combined antiangiogenic and antitumor activity. ${ }^{36,37}$ In addition, EG00229 has been shown to elicit immune-modulatory activity by blocking the M2 shift in microglial cells. ${ }^{39}$ Based on our previous work showing that the VEGF/NRP1 axis can be a great target for $\mathrm{CCRCC}^{16}$ we sought to examine whether EG00229 would have similar antitumor efficacy in RCC, either alone or in combination with other drugs.

Hence, in the present work, we proposed to target the mTOR pathway with Everolimus (E) and simultaneously disrupt the VEGFNRP1 axis by using EG00229 (G) that might block both VEGF/ VEGFR2/NRP1-mediated proangiogenic signaling pathways in endothelial cells and VEGF/NRP1/Ras-mediated autocrine activation of tumor cell growth. ${ }^{16}$ We assumed that by doing so, our strategy will reduce the amount of available VEGF and, at the same time, inactivate the residual VEGF from activating its proangiogenic and pro-tumorigenic downstream signaling pathways. Since both Everolimus and EG00229 are water-insoluble compounds, we developed a liposomal formulation entrapping both for easy and efficient delivery, instead of using complex delivery vehicles consisting of DMSO, ethanol, surfactants, or polyethylene glycol. Another added advantage of our liposomal formulation is that we have tagged a proprietary tumor-targeting peptide (TTP) to the surface of the liposomes to enhance the tumor-specific delivery of the drugs and reduce any toxicity arising from the treatment of Everolimus as well as systemic inhibition of NRP1. Here, we report the antitumorigenic and antimetastatic efficacy of the dual-drug-loaded liposomal formulation EG-L in two different $C C R C C$ xenograft models and the immunemodulatory effect of EG-L in a highly aggressive immunecompetent syngeneic mouse model of ccRCC.

\section{RESULTS}

Synthesis and characterization of liposomes

The amount of lipid and drug components of empty liposomes ( $L$ ) and drug-loaded liposomes (E-L, G-L, and EG-L) are reported in Table 1 along with drug-loading efficiency (DLE) and encapsulation efficiency (EE) values where applicable. The initial amounts of both Everolimus and EG00229 used during preparation of liposomes were $0.4 \mathrm{mg}$ per $1 \mathrm{~mL}$ of liposomes, respectively. Everolimus, being highly water-insoluble lipophilic drug, displayed an $\mathrm{EE}$ of $\sim 100 \%$ (concentration in liposome $=0.4 \mathrm{mg} / \mathrm{mL}$ ) due to its complete incorporation in the liposome bilayer. EG00229, initially being in the aqueous portion, displays only $30 \% \mathrm{EE}$ (concentration in liposome $=0.12 \mathrm{mg} / \mathrm{mL}$ ) due to its comparatively higher hydrophilic nature. The DLE of Everolimus in E- $\mathrm{L}$ and EG00229 in G-L were $7.29 \%$ and $2.2 \%$ respectively. The DLE and EE values in dual-drug-loaded liposomes (EG-L) did not show any alterations from the single drug-loaded ones. Plausibly, the distinct spatial distribution of Everolimus and EG00229 inside the liposomes is responsible for this observation. A higher DLE will require fewer amounts of carrier lipids to deliver the same amount of drugs thus increasing the efficacy of the treatments whereas a higher EE would ensure minimum loss of drugs during the preparation of the liposomal formulations. The DLE and EE of EG-L are more or less comparable with the values reported for the hydrophobic and hydrophilic drugs in literature. ${ }^{40}$

The average hydrodynamic size, polydispersity index (PDI), and zeta potential of empty liposomes $(\mathrm{L})$ as well as liposomes containing Everolimus (E-L), EG00229 (G-L), and a combination of both (EG-L) are consolidated in Table 2. The entrapment of drugs caused mostly minor changes in the size and PDI of the liposomes except for encapsulation of EG00229 (G-L) where the size and PDI of the liposomes increased significantly. Nonetheless, all the liposomal formulations had average size of less than $100 \mathrm{~nm}$ which is suitable for better penetration through tumor microenvironment. ${ }^{41}$ However, the zeta potentials were significantly different among the liposomes. The empty liposomes had a zeta potential of $32.9 \pm 2.3 \mathrm{mV}$ ( \pm values are given based on standard deviations, $n=3$ ). Encapsulation of Everolimus decreased the zeta potential to $18.3 \pm 1.7 \mathrm{mV}$ whereas encapsulation of EG00229 decreased it to $22.4 \pm 4.3 \mathrm{mV}$. The liposomes encapsulating both the drugs had a higher zeta potential $(47.53 \pm 1.6 \mathrm{mV})$ than other liposomes. A highly positive zeta potential indicates stability of the liposomal suspension as well as stronger interaction with negatively charged cell membranes. ${ }^{42}$ Since all of our liposomes were positively charged, we expect these formulations to be stable and efficient in cellular uptake.

In vivo biodistribution of liposomes in $\mathrm{ccRCC}$ xenograft bearing mice

After performing the physical characterizations, we decided to examine the in vivo tumor-targeting efficacy of these liposomes in ccRCC tumor bearing mice. Hence, we performed biodistribution studies in subcutaneous $786-\mathrm{O}$ or A498 xenografts after intravenous administration of IR-780-dye labelled liposomes. We used both TTP-conjugated liposomes (TL) and control liposomes without any TTP (CL) in these experiments. IR-780-dye was used as its excitation and emission peaks reside in the $I R$ region of the spectrum resulting minimal loss of intensity from absorption by live tissue. In addition, no discernible autofluorescence from mouse fur interfering with the signal intensity was witnessed in this region. As expected, TL resulted in higher tumor-specific signals than $\mathrm{CL}$ at 24 and $48 \mathrm{~h}$ post administration in both 786-O and A498 xenografts (Fig. 1a, b), which was further supported by the ex vivo imaging of the tumors and major organs (Fig. 1c, d). In addition, lungs from CL-treated mice displayed stronger signal than lungs of TL-treated mice, which indicates that TTP is more effective in diminishing the nonspecific accrual of the liposomes in the lungs.

In vitro efficacy of drug-loaded liposomes in ccRCC

On the basis of the above biodistribution study, we used TL in all further efficacy studies since our goal was to exploit the tumortargeting ability of TL for precision therapy. However, before going for animal studies, we examined the drug-loaded liposomal formulations for their in vitro efficacy in 786-O and A498 cells. As shown in Fig. 2a, b, EG-L was more effective in reducing cell viability than $\mathrm{E}-\mathrm{L}$ or $\mathrm{G}-\mathrm{L}$ in both $786-\mathrm{O}$ and A498 cell lines.

In vivo efficacy of drug-loaded liposomes in ccRCC xenografts We then proceeded to analyze the in vivo efficacy of the drugloaded liposomes in two different ccRCC xenografts in immunedeficient mouse models and one immune-competent syngeneic mouse model. In the initial screening experiments, we employed the single mouse trial (SMT) strategy, a lately popularized concept that has been well accepted by scientific community. ${ }^{43-45} \mathrm{~A}$ single mouse per treatment arm is employed in this approach to reliably detect the most effective treatment from a large number of treatment regimens in a cost-effective manner by analyzing the longitudinal growth of each tumor. Understandably, SMT is not expected to provide the statistical significance of the observed result; however, this limitation may be alleviated by performing a validation study in larger cohorts with the most effective treatment.

In our experiments, we allowed the tumors to grow larger before starting the treatment to challenge our drug-loaded liposomal formulations against comparatively worse pathological conditions. In most of our experimental design, a starting tumor volume of $300-500 \mathrm{~mm}^{3}$ has been used, which is considerably 
Table 1. Encapsulation efficiency (EE) and drug loading efficiency (DLE) of the liposomes.

\begin{tabular}{|c|c|c|c|c|c|c|c|c|}
\hline Liposome & $\begin{array}{l}\text { DOPC } \\
(\mathrm{mg} / \mathrm{mL})\end{array}$ & Cholesterol (mg/mL) & $\begin{array}{l}\text { DSPE(PEG)2000-OMe } \\
(\mathrm{mg} / \mathrm{mL})\end{array}$ & $\begin{array}{l}\text { TTP } \\
(\mathrm{mg} / \mathrm{mL})\end{array}$ & $\begin{array}{l}\mathrm{E} \\
(\mathrm{mg} / \mathrm{mL})\end{array}$ & $\mathrm{G}(\mathrm{mg} / \mathrm{mL})$ & DLE (\%) & EE (\%) \\
\hline L & 3.93 & 0.965 & 0.140 & 0.452 & - & - & - & - \\
\hline G-L & 3.93 & 0.965 & 0.140 & 0.452 & - & 0.120 & 2.2 & 30 \\
\hline EG-L & 3.93 & 0.965 & 0.140 & 0.452 & 0.4 & 0.120 & $7.29(\mathrm{E}), 2.2(\mathrm{G})$ & $100(\mathrm{E}), 30(\mathrm{G})$ \\
\hline
\end{tabular}

Encapsulation efficiency and drug loading efficiency of liposomes containing Everolimus (E-L), EG00229 (G-L), and combination of Everolimus and EG00229 (EG-L), $n=1$ measurement per sample

Table 2. Characterization of liposomal drug formulations.

\begin{tabular}{lcrr}
\hline Liposome & Size $(\mathrm{nm})$ & \multicolumn{1}{c}{ PDI } & Zeta $(\mathrm{mV})$ \\
\hline L & $67.1 \pm 0.12$ & $0.24 \pm 0.05$ & $32.9 \pm 2.3$ \\
E-L & $62.15 \pm 0.4$ & $0.18 \pm 0.01$ & $18.3 \pm 1.7$ \\
G-L & $95.35 \pm 0.69$ & $0.334 \pm 0.01$ & $22.4 \pm 4.3$ \\
EG-L & $71.86 \pm 0.16$ & $0.351 \pm 0.01$ & $47.53 \pm 1.6$
\end{tabular}

Hydrodynamic size, polydispersity index (PDI), and zeta potential of liposome only (L), or liposomes containing Everolimus (E-L), EG00229 (G-L), and a combination of Everolimus and EG00229 (EG-L). All the measurements were performed in deionized water at $25^{\circ} \mathrm{C} . \pm$ values are based on standard deviations, $n=3$ measurements per sample

higher than the usual $50-100 \mathrm{~mm}^{3}$ volumes commonly reported in tumor growth retardation studies. Similar to the in vitro studies, EG-L was better than E-L or G-L in impeding tumor growth in both 786-O and A498 xenografts (Fig. 2c, d). Importantly, EG-L demonstrated discernible reductions in tumor volumes from the initial higher values in both the tumor models, which suggests its superior antitumorigenic efficacy. This was further substantiated from the H\&E and Ki67 staining of the tumor sections (Fig. 3a-c) that exhibited significantly higher antiproliferative activity of EG-L than that of E-L or G-L. In addition, liver, kidney, and spleen did not show any significant changes in gross morphology, suggesting that those organs were not adversely affected by the drugloaded liposome treatment (Supplementary Figs S1 and 2).

We further performed a validation study in cohorts of five mice bearing 786-O xenografts to confirm whether the potent antitumorigenic efficacy of EG-L is truly reproducible and statistically significant. We obtained essentially similar results to the SMT demonstrating noticeable and statistically significant reductions in tumor volumes from higher starting values up to 3 weeks of treatment (Fig. 4a-d). In a way, this result corroborates the relevance of the SMT in recognizing the optimal treatment strategy for combating cancer.

We also analyzed the efficacy of EG-L in a highly aggressive syngeneic mouse ccRCC model developed by subcutaneous implantation of Renca cells in immune-competent Balb/c mice. This ccRCC model is resistant to immune therapy by anti-PD-1 antibody or small-molecule inhibitor of PD-1/PD-L1 interaction (Supplementary Fig. S3A, B) and therefore mimics $~ 80 \%$ of cCRCC patients who do not respond to immune therapy. Although EG-L was not able to reduce the tumor volume from initial value in this highly aggressive model, it displayed remarkable tumor growth retardation over the course of the study (Fig. 5a, b). The H\&E and Ki67 staining of the tumor sections demonstrated strong antiproliferative activity (Fig. 5c, d). In addition, a marked reduction in YM1 positivity in EG-L-treated tumor sections was observed (Fig. 5c, e). YM1 is a marker of macrophage M2 polarization, which is usually responsible for suppression of

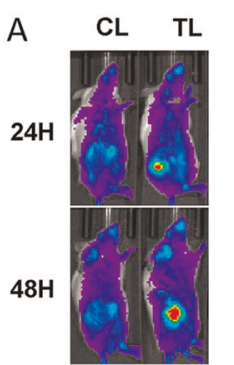

C

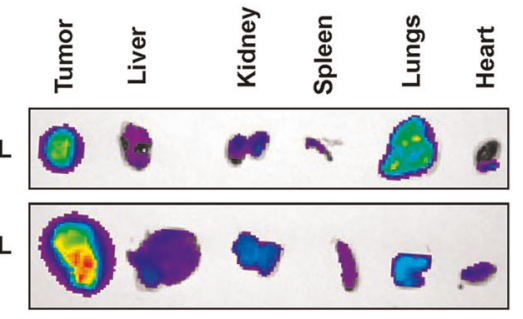

B

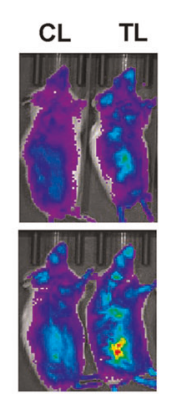

D

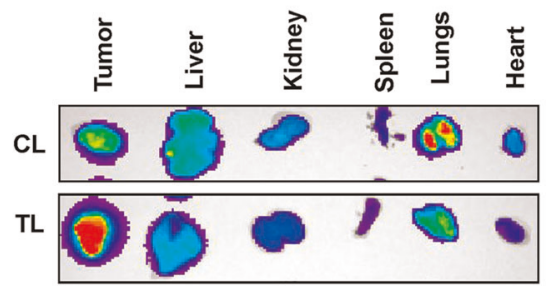

Fig. 1 In vivo biodistribution of IR-780-dye-labeled liposomes in RCC xenografts. IVIS imaging showing higher tumor accumulation of IR-780 dye-labeled TTP-conjugated liposomes (TL) compared to control liposomes $(\mathrm{CL})$ at $24 \mathrm{~h}$ (upper panel) and $48 \mathrm{~h}$ (lower panel) after IV administration into mice bearing subcutaneous 786-O (a) and A498 tumors (b). Ex vivo imaging of 786-O (c) and A498 (d) tumors and major organs, respectively, harvested at $48 \mathrm{~h}$, demonstrated significant higher tumor uptake of TL compared to CL. Interestingly, significantly higher lung accumulation of $\mathrm{CL}$ was observed compared to TL. $n=1$ mouse per treatment group.

antitumor immunity and increase in tumor growth. Hence, there may be a plausible immune-modulatory role of EG-L behind its strong antitumor response in this model. Interestingly, we did not observe any additive or synergistic effect when EG-L was combined with anti-PD-1 antibody or small-molecule inhibitor of PD-1/PD-L1 interaction (Supplementary Fig. S3A, B). Furthermore, we observed no significant change in the expression of PD-L1 or PD-1 in EG-L-treated tumors compared to control although PD-1 expression was slightly higher in the EG-L-treated group (Supplementary Fig. S4A-D). The uncropped scans of the blots are provided in Supplementary Fig. S5.

We also tested if EG-L treatment led to any alterations in chemokine and cytokine expression that are known modulators of immune infiltration. As depicted in Fig. 6, we observed significant reductions in mRNA levels of $\mathrm{C}-\mathrm{X}-\mathrm{C}$ motif chemokine receptor 3 (CXCR3)-associated chemokines C-X-C Motif Chemokine Ligand-9 (CXCL9), C-X-C Motif Chemokine Ligand-10 (CXCL10), and C-X-C Motif Chemokine Ligand-11 (CXCL11). TGFB1 mRNA levels were reduced as well, which corroborates with previous studies. ${ }^{38}$ 
A

786-0

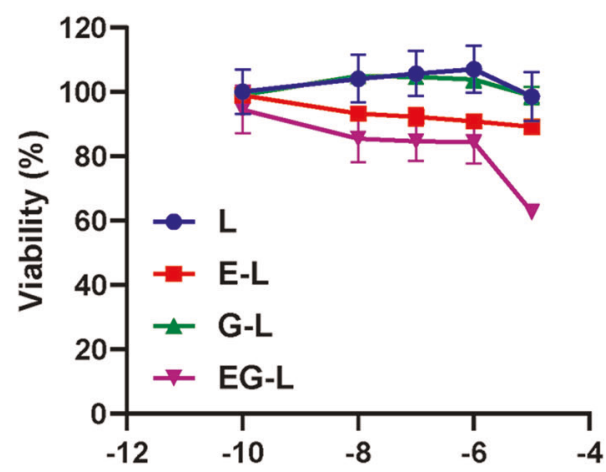

Liposome concentration (LogM)
B

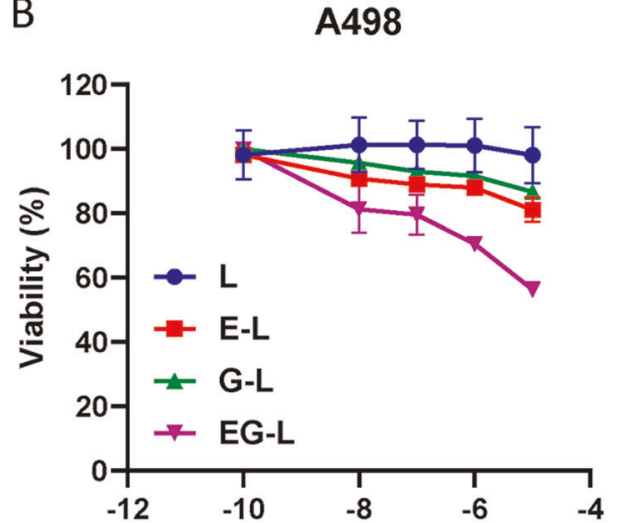

Liposome concentration (LogM)
C

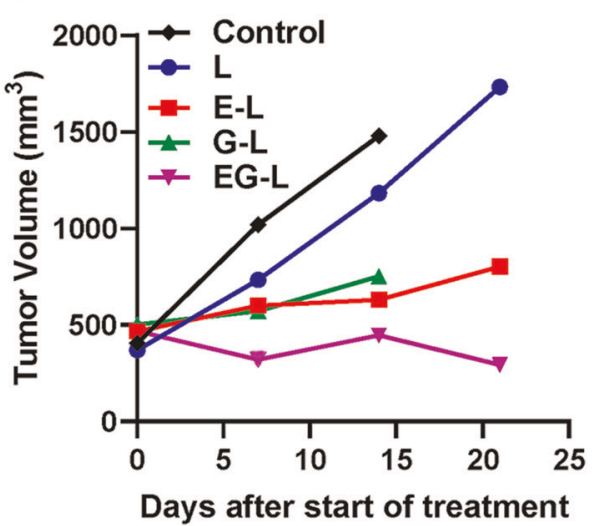

D

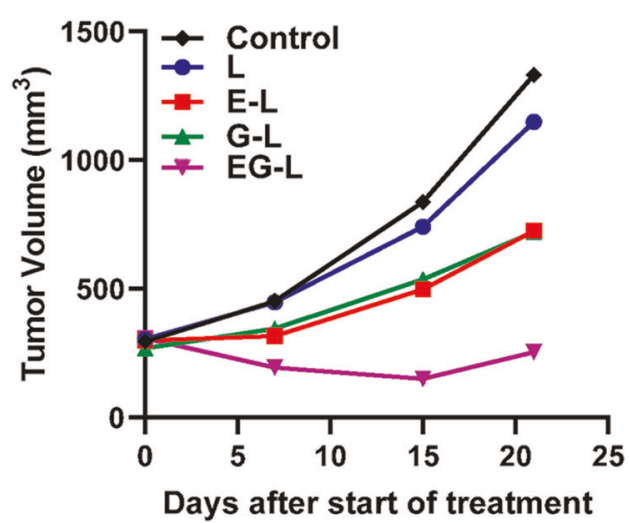

Fig. 2 In vitro and in vivo efficacy of drug-loaded liposomes in RCC cell lines. 786-O (a) and A498 (b) cells were treated with various drugloaded TTP-conjugated liposomes for $72 \mathrm{~h}$. Then cell viability was determined with MTS assay. Dual-drug-loaded liposomes showed higher reduction in cell viability compared to single drug-loaded liposomes in all cell lines ( $n=4$ wells per dose). c $5 \times 10^{6} 786-0$ cells were subcutaneously injected into the right flanks of 8-week-old male SCID mice. Tumors were allowed to grow until the average tumor size is $\sim 400-500 \mathrm{~mm}^{3}$. Then mice were treated with drug-loaded liposomes ( $n=1$ mouse per treatment group) $3 \times$ per week for 3 weeks. Tumors were measured weekly and tumor volume was plotted to obtain the respective growth curves. In both cases dual-drug-loaded liposomes demonstrated stronger inhibition compared to single drug-loaded liposomes. Some of the mice were sacrificed before the completion of experiment due to ulceration of tumors. d Similar results were obtained in A498 xenografts ( $n=1$ mouse per treatment group).

Inhibition of lung metastases

Since ccRCC is notorious for inducing lung metastases, ${ }^{46}$ we further explored the efficacy of our drug-loaded liposomal formulations in reducing the metastatic burden. Interestingly, the $\mathrm{H} \& \mathrm{E}$-stained whole-lung sections displayed metastatic nodules in the control mouse or mice treated with $\mathrm{L}$ or $\mathrm{E}-\mathrm{L}$, whereas $\mathrm{G}-\mathrm{L}$ and EG-L treated mice lung sections exhibited no detectable nodules (Fig. 7). Our results suggest that both G-L and EG-L were capable of reducing the lung metastases in $\mathrm{CcRCC}$, which might have significant clinical relevance.

\section{DISCUSSION}

Kidney cancer is the sixth and eighth most common cancer in men and women in the United States, respectively, and in 2019, an estimated 73,820 new diagnoses and 14,770 deaths are projected in the United States related to this desease. ${ }^{47}$ Approximately $90 \%$ of all kidney cancers are diagnosed as RCC. ${ }^{1}$ RCC has three major histologic subtypes: cCRCC (75-80\%), papillary RCC (10\%-16\%), and chromophobe RCC (5\%). ${ }^{2}$ While prognosis is usually better in patients with early stage RCC, advanced metastatic RCC is a life-threatening disease having a meagre $11.7 \% 5$-year survival rate. Approximately one-third of RCC patients have already developed metastasis at diagnosis and distant metastases is observed in up to $50 \%$ patients after resection of primary tumor. ${ }^{48}$ Conventional chemotherapy and radiation therapy are mostly futile in RCC. ${ }^{49,50}$ Current first- and second-line treatments for RCC consisting of tyrosine-kinase inhibitors (TKI) such as Sorafenib, Sunitinib, and Pazopanib; mTOR inhibitors such as Everolimus, Temsirolimus; and anti-VEGFhumanized antibody Bevacizumab failed to deliver long-term survival benefits. ${ }^{51-53}$ A recently introduced programmed death-1 (PD-1) antibody-based immune checkpoint inhibition therapy provided objective response in a subset of patients $(\sim 25 \%)$; however, the improvement in median overall survival is not drastically improved. ${ }^{54}$ Lately, a combination of two immune checkpoint inhibitors, Nivolumab and Ipilimumab, eclipsed the efficacy of Sunitinib in a phase III trial and has been approved as the new standard of care treatment for intermediate- and poorrisk patients with previously untreated advanced RCC. ${ }^{55}$ Moreover, the combination of Axitinib with either Avelumab (a programmed death ligand 1 inhibitor) or Pembrolizumab (a programmed death1 inhibitor) exhibited superior progression-free survival and objective response rate over Sunitinib in two distinct large phase III trials. ${ }^{56,57}$ Significant improvement in overall survival was also observed in patients treated with Axitinib and Pembrolizumab. Expectedly, both the combinations have been approved for the first-line treatment in advanced RCC. However, treatment-related 


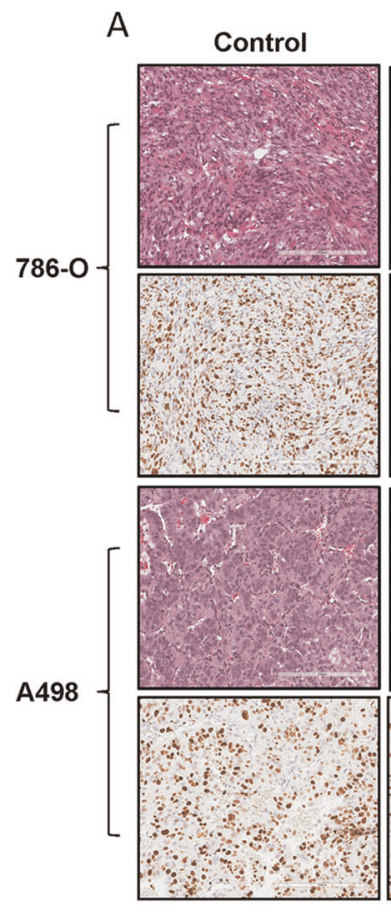

B
L

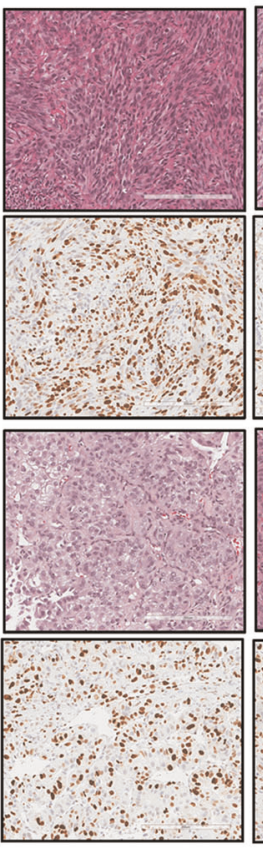

786-0

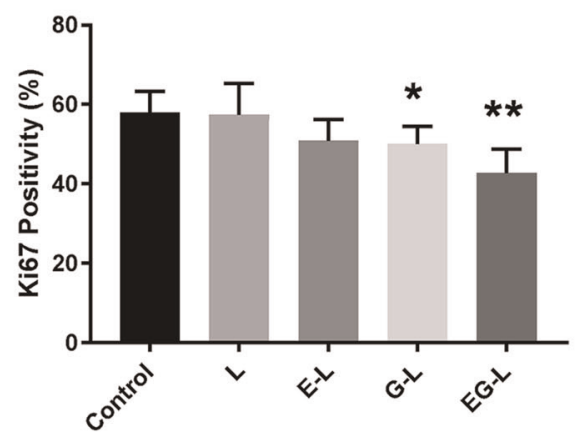

E-L

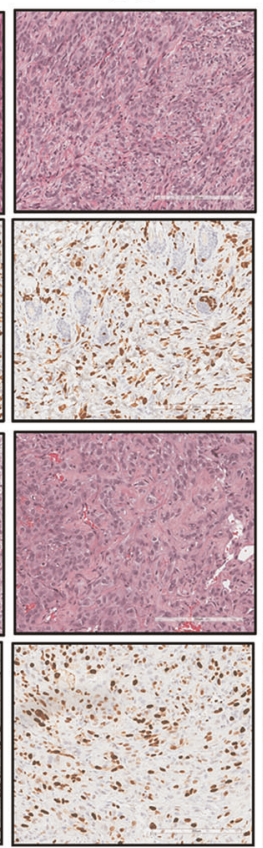

C

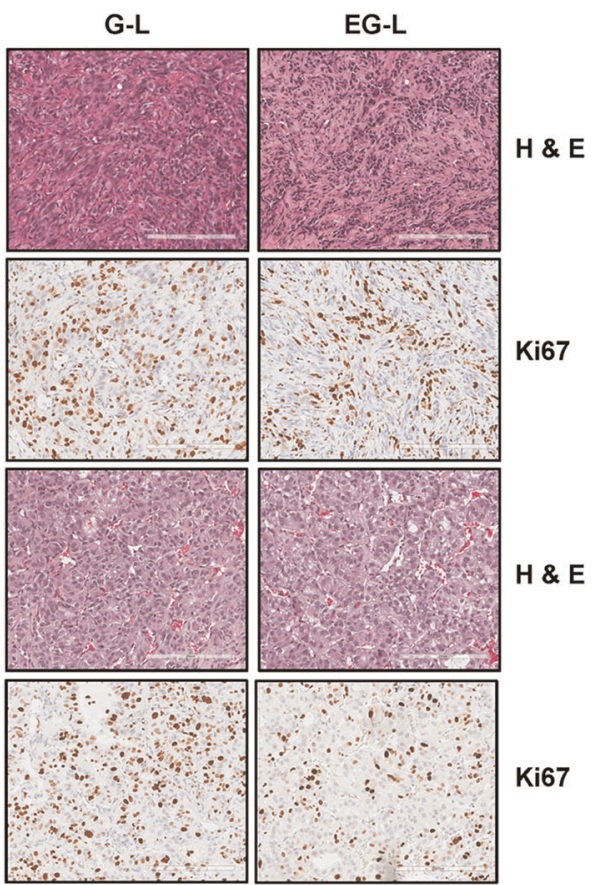

A498

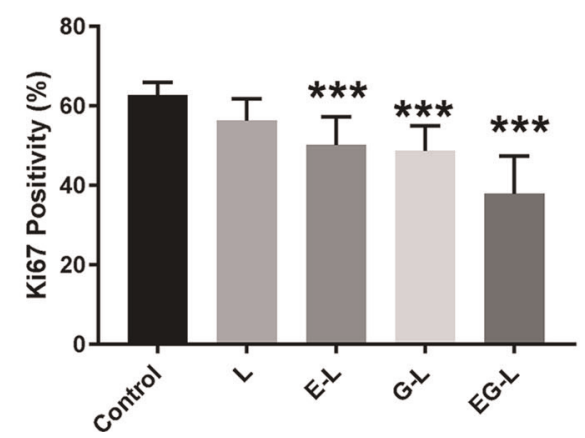

Fig. $3 \mathrm{H} \& \mathrm{E}$ and Ki67 staining of tumor sections obtained from the single mouse trial. a Representative images of the H\&E and Ki67 stained tumor sections from different treatment groups displayed comparatively higher antiproliferative activity of EG-L. Bar length $=200 \mu \mathrm{m}$. $\mathbf{b}$, $\mathbf{c}$ Quantification of Ki67-positive nuclei in 786-O and A498 tumor sections, respectively. Error bars in all graphical plots are given based on standard deviation. ${ }^{*}, * *$ and ${ }^{* * *}$ denote $p<0.05, p<0.01$, and $p<0.001$ compared to control, respectively ( $n=5$ spatially different regions from same tumor section).

adverse events were observed in a majority of patients treated with these combination therapies. Therefore, an unmet clinical need exists for novel treatment strategies as well as targeted delivery systems for advanced metastatic RCC.

The phosphatidyl-inositol-3 kinase (PI3K)/Akt/mTOR pathway is a signaling axis responsible for various crucial functions in cellular homeostasis including protein synthesis, glucose metabolism, survival, migration, and angiogenesis. ${ }^{5,59}$ It has also been implicated in the pathogenesis of various cancers including RCC. $^{60}$ mTOR inhibitors such as Temsirolimus and Everolimus have been approved for first- and second-line treatments for RCC, respectively. We selected Everolimus in our study since it was approved for second- and third-line therapy in patients with advanced RCC. Previously, Everolimus had been shown to inhibit cell growth, migration, and invasion in RCC cell lines in vitro. ${ }^{61,62}$ Everolimus manifested antiangiogenic properties as well, but in a different way than other VEGFR-TKIs. ${ }^{63}$ Everolimus has been shown to inhibit VEGF expression in tumor cells. ${ }^{61,64}$ Interestingly, no significant clinical benefit of Everolimus was found in first-line setting either as a monotherapy or in combination with Bevacizumab. ${ }^{65}$ However, a recent Phase II study demonstrated that a second-line combination therapy with Everolimus and Lenvatinib (a novel TKI) commanded a substantial increase in progression-free survival and overall survival compared to monotherapy with Everolimus. ${ }^{66}$ Hence, it is prudent to postulate that a carefully designed combination strategy with Everolimus as one of the agents may prove to be beneficial.

Recently it has been proposed that the role of VEGF in cancer is not all about angiogenesis and vascular permeability. Several recent articles including ours confirmed the presence of a VEGFmediated autocrine signaling mechanism in tumor cells that contributes to tumorigenesis and drug resistance by imparting stem-cell like features to cancer cells. ${ }^{15-19}$ Interestingly, all these articles point towards the VEGF/NRP1 axis to be the major player behind this recently discovered angiogenesis-independent function of VEGF. As NRP1 lacks a kinase domain, downstream effector molecules including $\operatorname{Ras}^{16}$ and $\beta$-catenin ${ }^{18}$ have been proposed to be involved in this intriguing signaling pathway. Nonetheless, the VEGF/NRP signaling axis became a prime target overnight for anticancer therapy because it can impart stemness and drug resistance to cancer cells. 


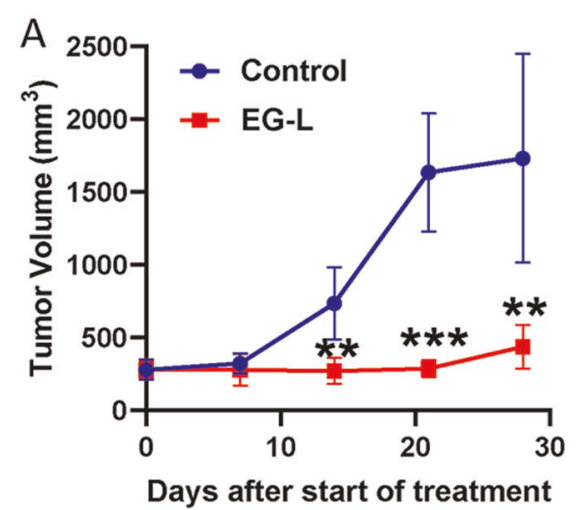

C

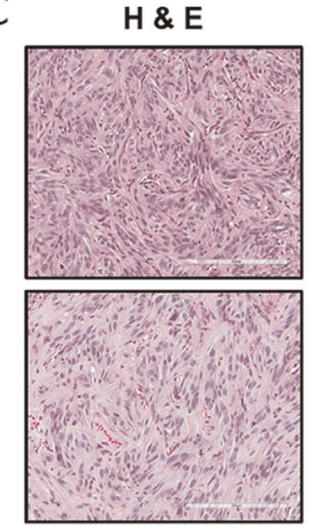

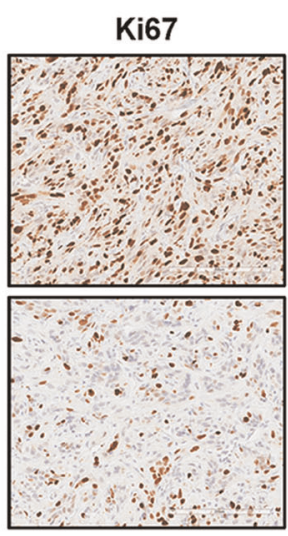

D

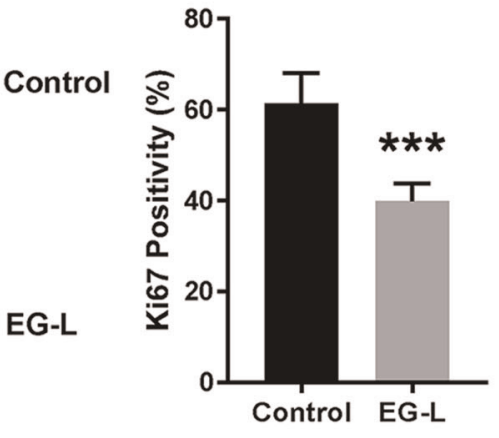

Fig. 4 Validation of the result obtained from single mouse trial in cohorts of five mice. a $5 \times 10^{6} 786-0$ cells were subcutaneously injected into the right flanks of 8-week-old male SCID mice. Tumors were allowed to grow until the average tumor size is $\sim 300 \mathrm{~mm}^{3}$. Then mice were treated with vehicle or EG-L ( $n=5$ mice per treatment group) $3 \times$ per week for 4 weeks. Tumors were measured weekly and tumor volume was plotted to obtain the respective growth curves. EG-L demonstrated significant inhibition compared to the vehicle group. ** and ${ }^{* * *}$ denote $p$ $<0.01$ and $p<0.001$ compared to control, respectively. b Images of the harvested tumors at the end of the experiment. c Representative images of H\&E and Ki67 staining of the tumor tissue sections. Bar length $=200 \mu \mathrm{m}$. d Quantification of Ki67-positive nuclei. ${ }^{* * *}$ denotes $p<$ 0.001 compared to control ( $n=3$ tumors per group, five spatially different regions from each tumor section).

A number of peptide-based competitive inhibitors of VEGFNRP1 binding were developed that were able to inhibit downstream VEGF signaling pathways. ${ }^{32-35}$ EG00229, the first smallmolecule competitive inhibitor of VEGF-NRP1 binding, ${ }^{36}$ has been designed from previously developed bicyclic peptide EG3287 that resembles the C-terminal of VEGF. ${ }^{34}$ EG00229 has been shown to bind to the b1 domain of NRP1 leading to the disruption of VEGFNRP1 binding. ${ }^{36}$ This, in turn, results in the inhibition of VEGF/ VEGFR2/NRP1-mediated proangiogenic signaling in endothelial cells. In addition, disruption of VEGF-NRP1 axis inhibits the tumor cell autocrine signaling via regulating the expression and function of various downstream effector molecules including Ras and $\beta$-catenin. ${ }^{16,18}$ Therefore, it is not at all surprising that EG00229 demonstrates combined antiangiogenic and antitumor activity to delay tumor progression in multiple cancer models. ${ }^{18,36,37}$ Moreover, the immune-modulatory function of EG00229 has been suggested in a recent article. ${ }^{39}$

Hence, in the present work, we hypothesized that targeting the VEGF signaling in $\mathrm{CCRCC}$ via a bifurcated approach by combining Everolimus and EG00229 in a tumor-targeted liposomal formulation will be beneficial. In addition to its antiproliferative effect, Everolimus will inhibit VEGF synthesis, thereby reducing VEGF levels in tumor microenvironment. At the same time, EG00229 will disrupt VEGF-NRP1 axis leading to the inhibition of VEGF/VEGFR2/ NRP1-mediated proangiogenic signaling pathways in endothelial cells and VEGF/NRP1/Ras-mediated autocrine activation of tumor cell growth. Therefore, our strategy enjoys a significant difference from the combination of Everolimus and Bevacizumab that did not improve the patient outcome remarkably. Bevacizumab works by binding to VEGF that is secreted in the tumor microenvironment and inhibiting its angiogenic activity. On the contrary, EG00229 binds to the b1 domain of NRP1 and disrupts the angiogenic signaling in tumor-endothelial cells as well as autocrine signaling in tumor cells as mentioned above. In addition, immune-modulatory effect of EG00229 may act against the immune-suppressive function of Everolimus, ${ }^{67}$ thus promoting antitumor immunity. Consequently, the combination of Everolimus and EG00229 is anticipated to be superior to the combination of Everolimus and Bevacizumab, although this concept needs further evaluation.

We further postulated that the tumor-targeted delivery will require lower doses of Everolimus and EG00229. Indeed, we attained notable tumor inhibition while using lower doses of these drugs than are usually administered. We used $1 \mathrm{mg} / \mathrm{kg}$ Everolimus three times a week whereas it is normally administered daily via oral route at $1-5 \mathrm{mg} / \mathrm{kg} /$ day. ${ }^{68}$ Similarly the typical dose of EG00229 is $10 \mathrm{mg} / \mathrm{kg}$ three times a week via intraperitoneal route $^{19}$ but we have only used $300 \mu \mathrm{g} / \mathrm{kg}$ EG00229 three times a week. Of importance, we only doubled the dose for the highly aggressive Renca model but decreased the frequency of administration. We started the treatments with larger initial tumor volumes than those typically used in the animal studies reported in literature and still achieved significant tumor growth inhibition. We also demonstrated the immune-modulatory effect of EG-L in an immune-competent mouse model of cCRCC. CXCR3-associated chemokine ligands CXCL9, CXCL10, and CXCL11 demonstrate pleotropic roles in immunity and angiogenesis and correlate with poor prognosis in patients with ccRCC. ${ }^{69,70}$ Increased expressions 
A

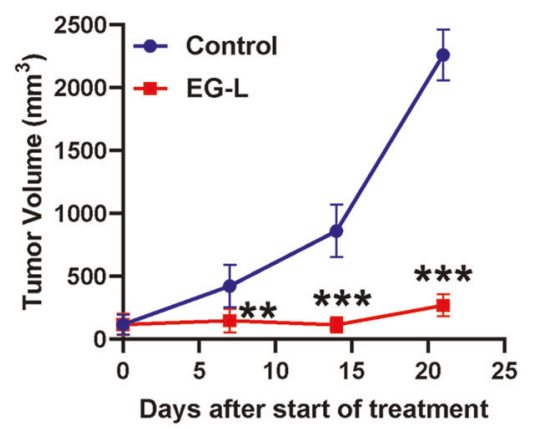

B

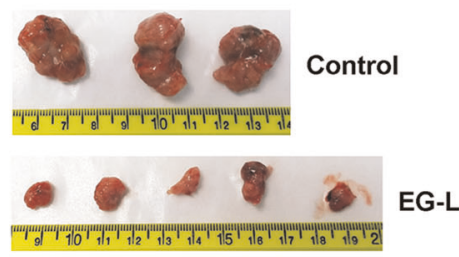

$\mathrm{C}$

H \& E
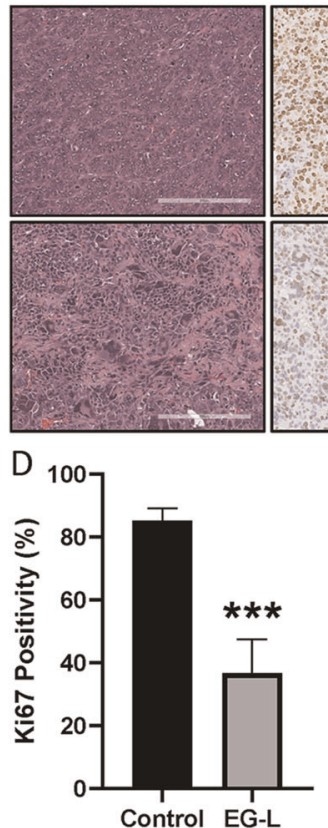

Ki67
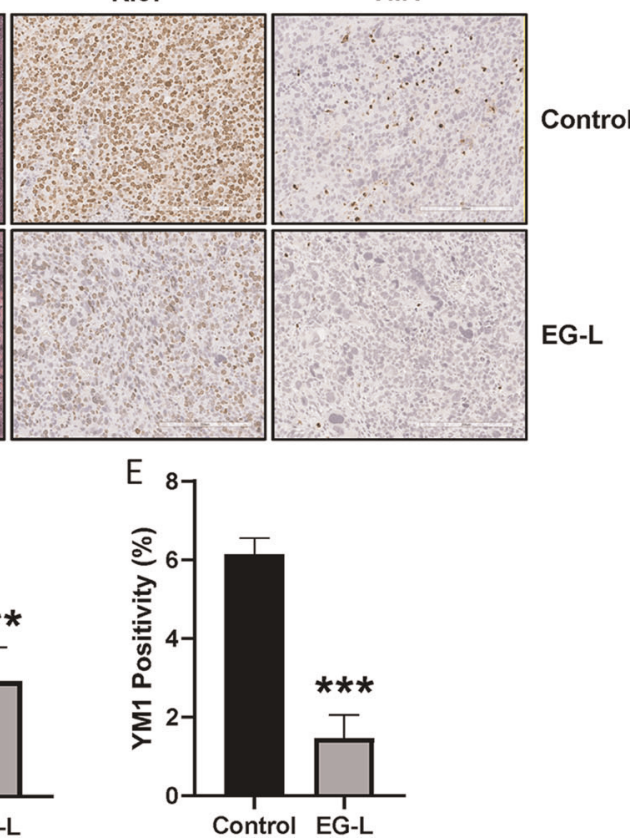

Fig. 5 Antitumor efficacy of EG-L in an immune-competent mice model of RCC. a $1 \times 10^{6}$ Renca cells were subcutaneously injected into the right flanks of 8 week-old-male Balb/c mice. Tumors were allowed to grow until the average tumor size is $\sim 120 \mathrm{~mm}^{3}$. Then mice were treated with vehicle or EG-L ( $n=5$ mice per treatment group) $2 \times$ per week for 3 weeks. Tumors were measured weekly and tumor volume was plotted to obtain the respective growth curves. EG-L demonstrated significant inhibition compared to the vehicle group. ${ }^{* *}$ and ${ }^{* * *}$ denote $p<0.01$ and $p<0.001$ compared to control, respectively. $\mathbf{b}$ Images of the harvested tumors at the end of the experiment. Two tumors from control groups were ruptured before harvest. c Representative images of H\&E, Ki67, and YM1 staining of the tumor tissue sections. Bar length $=$ $200 \mu \mathrm{m}$. d, e Quantification of Ki67 and YM1 staining respectively. ${ }^{* * *}$ denotes $p<0.001$ compared to control ( $n=3$ tumors for control and 4 tumors for EG-L, five spatially different regions from each tumor section).

of these chemokines were observed in tumors compared to the normal kidney tissues. ${ }^{71}$ These CXCR3 ligands are also known to recruit regulatory $T$ cells that lead to the suppression of effector $\mathrm{T}$ cells which may be one of the reasons of the poor patient outcome. ${ }^{72-74}$ In addition, increased expression of these chemokines has been associated with metastasis in RCC. ${ }^{75}$ Hence, these ligands exert a paracrine effect on tumor microenvironment as well as an autocrine effect in the tumor cells. Similarly TGF- $\beta$ signaling has been implicated in RCC progression and metastasis. ${ }^{76,77}$ Hence, by reducing the levels of these ligands, EG-L may improve the therapeutic response in patients. Finally, our treatment strategy demonstrated promising reductions in the metastatic burden that may prove to be beneficial for ameliorating the poor survival in patients with advanced metastatic ccRCC.

In summary, we demonstrated that simultaneous tumortargeted inhibition of mTOR (with Everolimus) and VEGF/NRP1 axis (with EG00229) with the help of a tumor-targeting liposomal formulation was able to induce remarkable inhibition of tumor growth in two different ccRCC xenografts and in a highly aggressive syngeneic mouse model of kidney cancer. In addition, this treatment regimen substantially inhibited the lung metastasis. Taken together, our data establish that a tumor-targeted liposomal formulation encapsulating Everolimus and EG00229 may offer a prospective therapeutic option towards the arsenal of currently available treatment regimens for combating metastatic ccRCC.

\section{METHODS}

\section{Reagents}

DOPC and DSPE-(PEG) 2000 -OMe were purchased from Avanti Polar Lipids and Nanosoft Polymers, respectively. Cholesterol was purchased from Sigma. Everolimus and EG00229 were obtained from LC laboratories and
Tocris Bioscience, respectively. Ki67 (ab15880), PD-L1 (PA5-88105), $\beta$-actin (A2228), and YM1 (60130) antibodies were purchased from Abcam, Invitrogen, Sigma, and STEMCELL Technologies, respectively. The goatanti-mouse PD-1 antibody was a kind gift from Dr. Keith L. Knutson (Mayo Clinic).

\section{Cell culture}

786-O and A498 cell lines were obtained from American Type Culture Collection (ATCC). Renca cell line was a kind gift from Dr. John A. Copland (Mayo Clinic). No authentication of the cell lines was done by the authors. 786-O and A98 cell lines were maintained in Dulbecco's modified Eagle's medium (DMEM) and RPMI-1640 medium was used for maintaining Renca cell line. Both the media were supplemented with $10 \%$ FBS and $1 \%$ penicillin-streptomycin (Invitrogen) and cells were cultured at $37^{\circ} \mathrm{C}$ in a humidified atmosphere with $5 \% \mathrm{CO}_{2}$. Cells from $85 \%$ to $90 \%$ confluent cultures were used in all of the experiments.

\section{Synthesis of tumor-targeting-peptide (TTP)-conjugated} lipopeptide

Fmoc-strategy-based solid phase peptide synthesis method was used to synthesize the TTP (a tumor-targeting-peptide with a proprietary sequence)-conjugated lipopeptide used in this study. ${ }^{78}$

\section{Preparation of empty liposomes}

A modified ethanol injection method was used to prepare the liposomes. ${ }^{78}$ Required amounts (Table 1) of TTP-conjugated lipopeptide, phospholipids, and cholesterol were dissolved in ethanol and the solution was warmed at $65^{\circ} \mathrm{C}$ for $5 \mathrm{~min}$. The solution was then slowly injected into milli-Q water pre-heated to $65^{\circ} \mathrm{C}$ while stirring the mixture continuously which led to the spontaneous formation of liposomes. The liposomal solution was stirred at room temperature for an additional $15 \mathrm{~min}$. Finally, rotary evaporation was used to remove ethanol and a part of water under reduced pressure and volume was adjusted with milli-Q water. The liposomes were stored at $4{ }^{\circ} \mathrm{C}$ until further use. 


\section{Preparation of drug-loaded liposomes}

To prepare the dual-drug-loaded liposomes, EG00229 and Everolimus were included in the aqueous phase and the ethanolic solution of lipids, respectively. The single drug-loaded liposomes were prepared via similar methods where only the drug of choice was used. Following removal of ethanol, Amicon ultra centrifugal filters ( $3 \mathrm{kDa}$ molecular weight cut-off) were used to remove any unentrapped drugs. The liposome concentrates thus obtained were diluted back to original volumes with milli-Q water and were stored at $4{ }^{\circ} \mathrm{C}$ until further use.
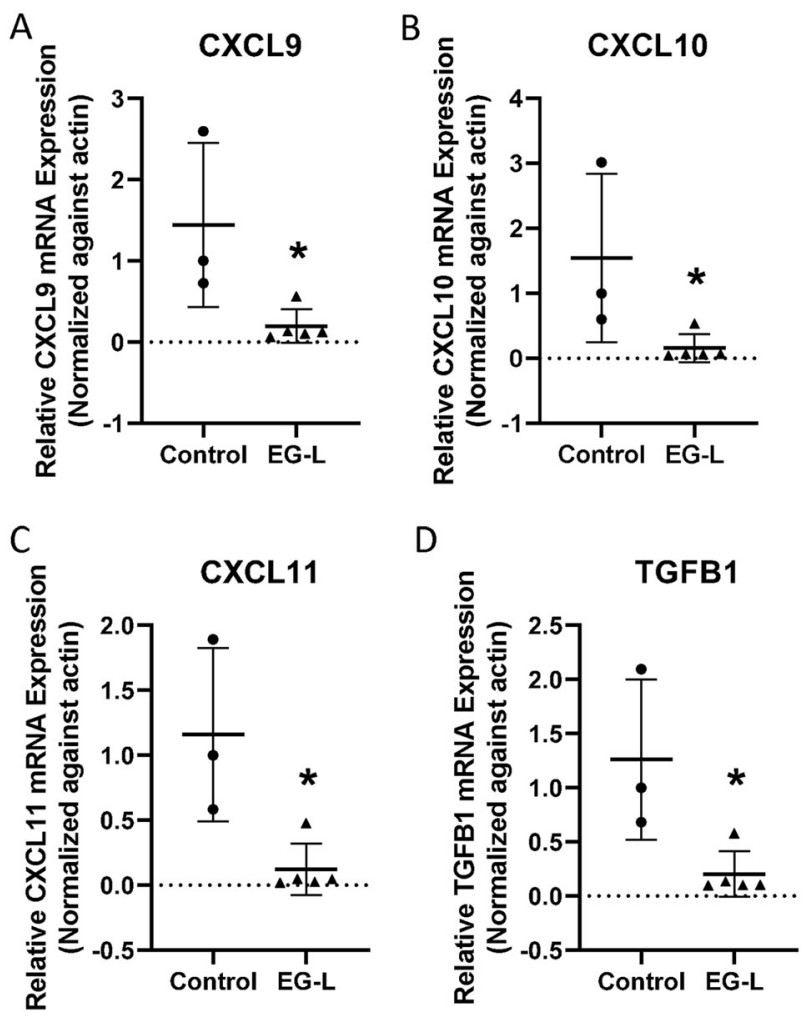

Fig. 6 EG-L downregulates the expression of C-X-C chemokines and TGF- $\beta$ in an immune-competent mice model of RCC. Total RNA was isolated from tumors treated with vehicle or EG-L and subjected to real-time reverse transcription polymerase chain reaction (RT-PCR) for a CXCL9, b CXCL10, c CXCL11, and d TGFB1. EG-L significantly reduced the mRNA expression of the cytokines compared to the vehicle. * denotes $p<0.5$ compared to control. ( $n=3$ tumors for control and 5 tumors for EG-L).
Liposome size and zeta potential analysis

Dynamic light scattering (DLS) measurements were performed using a Malvern Zetasizer (Malvern, UK) to determine the mean hydrodynamic diameter and zeta potential of empty and drug-loaded liposomes after sample dilution with deionized water. Triplicate measurements were performed in samples diluted with milli-Q water at $25^{\circ} \mathrm{C}$.

\section{Analysis of DLE and EE}

EE and DLE were calculated by estimating the amount of entrapped drugs according to previously published procedures. Briefly, during preparation of drug-loaded liposomes, the unentrapped drugs $\left(U_{E_{\text {drug }}}\right)$ were collected in the filtrate obtained from the amicon ultra centrifugal filter with a cut-off size of $3 \mathrm{kDa}$. Then, absorbance values at $\lambda_{\max }$ of respective drugs in the filtrates were measured and compared with respective standard curves to determine $\mathrm{UE}_{\text {drug }}$ amounts. The encapsulated drug $\left(\mathrm{E}_{\text {drug }}\right)$ amount was obtained by deducting $\mathrm{UE}_{\text {drug }}$ from total drug $\left(\mathrm{T}_{\text {drug }}\right)$ amount. Finally, $\mathrm{EE}$ was calculated as the percentage of $E_{\text {drug }}$ to $T_{\text {drug }}$ while DLE was expressed as the percentage of $E_{d r u g}$ to the total lipid amount ( $T_{\text {lipid }}$ ).

\section{Animals used in the study}

Six- to eight-week-old male SCID and Balb/c mice were obtained from inhouse breeding and housed in the institutional animal facilities. All animal experiments described in this study were performed under Mayo Clinic Institutional Animal Care and Use Committee (IACUC) approved protocols.

\section{In vivo biodistribution of liposomes}

$5 \times 10^{6} 786-\mathrm{O}$ or A498 cells resuspended in $100 \mu \mathrm{L}$ of $50 \%$ matrigel in PBS were subcutaneously implanted into the right flank of six- to eight-weekold male SCID mice. After 6-7 weeks, when the average size of tumors reached $300-500 \mathrm{~mm}^{3}$, either control (CL) or TTP-conjugated (TL) liposomes loaded with IR-780-Dye were intravenously administered. Fluorescence imaging was performed in live mice under anesthesia using an IVIS imager 24 and $48 \mathrm{~h}$ post administration. Finally, mice were euthanized to harvest the tumors and major organs for ex vivo imaging.

\section{In vitro cytotoxicity assay}

Approximately, $5 \times 10^{3} 786-\mathrm{O}$ or A498 cells per well were seeded in 96-well plates and allowed to settle for $18-24 \mathrm{~h}$. Then, cells were treated with increasing concentrations of $\mathrm{L}, \mathrm{E}-\mathrm{L}, \mathrm{G}-\mathrm{L}$, and EG-L diluted in respective media and incubated for an additional $72 \mathrm{~h}$ ( $n=4$ wells per concentration). Cell viability was determined with Celltiter 96 Aqueous One Solution Cell Proliferation Assay kit (Promega) following the manufacturer's protocol. Briefly, cells were washed once with PBS after aspirating the media containing the treatments from the wells. Then $100 \mu \mathrm{L}$ fresh media supplemented with $20 \%$ One Solution reagent was added to each well and the plate was incubated for $30 \mathrm{~min}$ at $37^{\circ} \mathrm{C}$. Finally, absorbance at $492 \mathrm{~nm}$ was determined using Spectramax i $3 x$. Percentage viability is calculated using the formula: viability $(\%)=100 \times\left(A_{\text {Treated }}-A_{\text {Blank }}\right) /\left(A_{\text {Untreated }}-A_{\text {Blank }}\right)$.
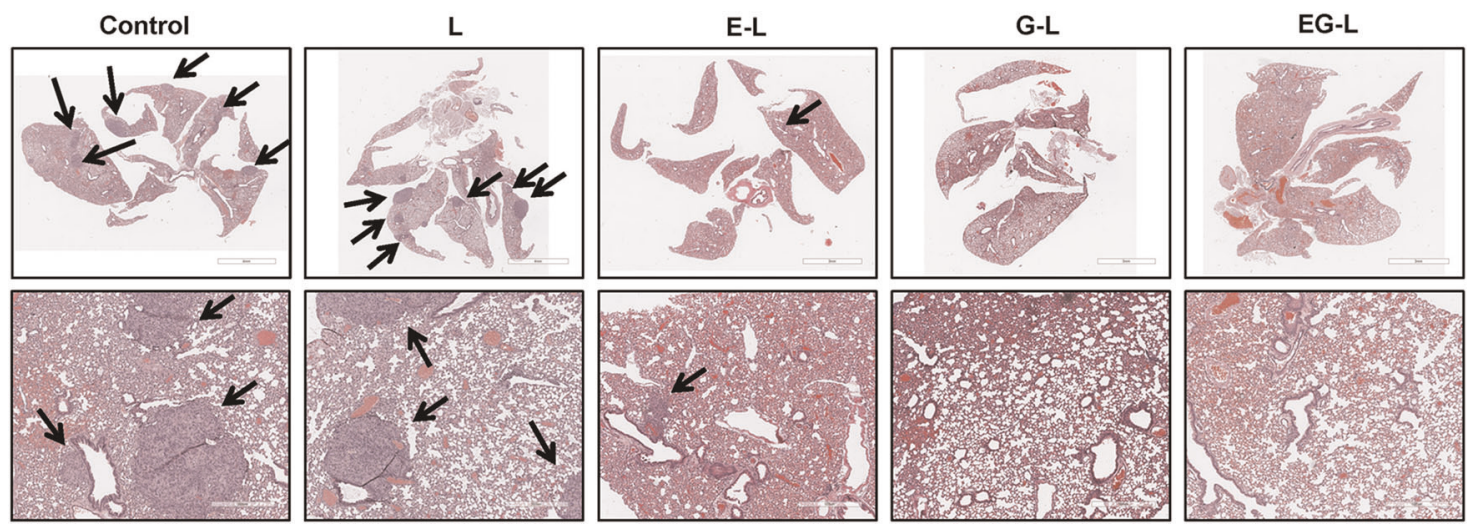

Fig. 7 Inhibition of lung metastasis in mice bearing 786-O xenografts. The dual-drug-loaded liposomes (EG-L) significantly inhibited lung metastasis in mice bearing 786-O subcutaneous tumors compared to Control, liposome only (L), or single-drug-loaded liposomes (E-L and GL). Metastatic nodules are indicated by black arrows (upper panel). Respective higher magnification images are depicted in the lower panel ( $n$ $=1$ mouse per treatment group). 


\section{In vivo tumor regression experiment}

The in vivo tumor regression efficacy of the drug-loaded liposomes was analyzed in an SMT using subcutaneous $786-0$ xenografts $(n=1$ per treatment group). Empty liposome (L), liposome containing Everolimus ( $\mathrm{E}-$ L), EG00229 (G-L), and a combination thereof (EG-L) were intravenously administered three times a week into mice bearing $~ 300-500 \mathrm{~mm}^{3}$ tumors. The liposome amounts for each treatment was determined to keep the Everolimus amount administered in E-L and EG-L treated groups constant at $20 \mu \mathrm{g} / \mathrm{mouse} /$ dose. Tumors were measured weekly with calipers and tumor volumes were calculated using the formula: volume $=0.5 \times a \times b^{2}$ where $a$ and $b$ are the longest and shortest diameter, respectively. Tumor growth curves were obtained by plotting tumor volumes against time. Finally, mice were sacrificed to harvest the tumors along with liver, kidney, and spleen for immunohistochemistry. A similar experiment was performed in A498 xenografts ( $n=1$ per treatment group). In order to validate the results obtained from the SMT, we analyzed the efficacy of EG$\mathrm{L}$ in larger cohorts of 786-O tumor bearing mice $(n=5$ per treatment group). In addition, we also analyzed the efficacy of EG-L in Renca syngeneic mouse $c c R C C$ model in Balb/c mice ( $n=5$ per treatment group), a highly aggressive tumor that accurately mimics the growth pattern of human CCRCC. Due to the aggressive tumor growth, we started treatment at $\sim 120 \mathrm{~mm}^{3}$ starting tumor volume and increased the dose of Everolimus to $40 \mu \mathrm{g} / \mathrm{mouse} /$ dose but reduced the frequency of administration to twice a week in this experiment. In addition, anti-PD-1 antibody and a smallmolecule inhibitor of PD-1/PD-L1 interaction were used in two separate SMT experiments ( $n=1$ per treatment group) in the Renca model to analyze any additive or synergistic effect on EG-L treatment.

\section{Immunohistochemistry}

Tumors, livers, kidneys, and spleens were harvested and fixed in neutralbuffered $10 \%$ formalin at room temperature for $24 \mathrm{~h}$. Then they were embedded in paraffin and 5 - $\mu \mathrm{m}$-thick sections were cut for preparing slides. Hematoxylin and eosin (H\&E) and Ki67 staining (1:1000) were performed in deparaffinaized slides as per the manufacturer's instructions (DAB 150; Millipore). For Renca tumor sections, YM1 immunostaining (1:100) was also performed. Slides were stained with stable diaminobenzidine and counterstained with hematoxylin. Finally, slides were digitized using an Aperio AT2 slide scanner (Leica) and analyzed using Imagescope software (Leica).

\section{Quantitative polymerase chain reaction (qPCR)}

Total RNA was isolated from a portion of the tumors using RNeasy Plus Mini Kit (Qiagen) as per the manufacturer's instructions. Reverse transcription was performed using iScript ${ }^{\mathrm{TM}}$ CDNA Synthesis Kit (Bio-Rad). Primers were designed using Ensembl genome browser 96 (Supplementary Table S1). Finally, qPCR was performed for the specified targets using Power SYBR Green PCR Master Mix (Applied Bioscience) in an ABI 7500 Real-Time PCR System (Applied Bioscience).

\section{Immunoblot analysis}

Lysates were prepared from homogenized tumor samples using NP-40 lysis buffer supplemented with a protease inhibitor cocktail. Protein concentrations of the lysates were measured by Bradford assay. An equal amount of proteins from each sample was subjected to SDS-PAGE and transferred to polyvinyl difluoride membranes followed by immunoblotting with PD-L1 (1:1000), PD-1 (1:500), and $\beta$-actin (1:10,000) antibodies and respective secondary antibodies $(1: 10,000)$. Enzyme-linked chemiluminescence was used to detect antibody-reactive bands in Chemidoc MP (Bio-Rad). Quantification of band intensities was performed using Image Lab (Bio-Rad). Blots from same experiments were used for presentation. The uncropped scans of the blots are provided in Supplementary Fig. S5.

\section{Statistical methods}

The double-sided unpaired two-tailed $t$-test was utilized to determine the probability of significant differences between treatment groups where applicable. Statistical significance was defined as ${ }^{*} P<0.05,{ }^{*} P<0.01$, and ${ }^{* * *} P<0.001$, respectively. Error bars are indicative of calculated SD values.

\section{DATA AVAILABILITY}

All data supporting the conclusions of this study are available within this article and the supplementary information.

Received: 15 April 2019; Accepted: 17 October 2019; Published online: 05 December 2019

\section{REFERENCES}

1. Low, G., Huang, G., Fu, W., Moloo, Z. \& Girgis, S. Review of renal cell carcinoma and its common subtypes in radiology. World J. Radiol. 8, 484-500 (2016).

2. Shuch, B. et al. Understanding pathologic variants of renal cell carcinoma: distilling therapeutic opportunities from biologic complexity. Eur. Urol. 67, 85-97 (2015).

3. Haase, V. H. The VHL tumor suppressor: master regulator of HIF. Curr. Pharm. Des. 15, 3895-3903 (2009).

4. Mukhopadhyay, D., Knebelmann, B., Cohen, H. T., Ananth, S. \& Sukhatme, V. P. The von Hippel-Lindau tumor suppressor gene product interacts with Sp1 to repress vascular endothelial growth factor promoter activity. Mol. Cell Biol. 17, 5629-5639 (1997).

5. Pal, S., Claffey, K. P., Dvorak, H. F. \& Mukhopadhyay, D. The von Hippel-Lindau gene product inhibits vascular permeability factor/vascular endothelial growth factor expression in renal cell carcinoma by blocking protein kinase $\mathrm{C}$ pathways. J. Biol. Chem. 272, 27509-27512 (1997).

6. Patard, J. J. et al. Absence of VHL gene alteration and high VEGF expression are associated with tumour aggressiveness and poor survival of renal-cell carcinoma. Br. J. Cancer 101, 1417-1424 (2009).

7. Dvorak, H. F., Brown, L. F., Detmar, M. \& Dvorak, A. M. Vascular permeability factor/vascular endothelial growth factor, microvascular hyperpermeability, and angiogenesis. Am. J. Pathol. 146, 1029-1039 (1995).

8. Gerber, H.-P. et al. Vascular endothelial growth factor regulates endothelial cell survival through the phosphatidylinositol 3'-kinase/Akt signal transduction pathway. J. Biol. Chem. 273, 30336-30343 (1998).

9. Watanabe, Y., Lee, S. W., Detmar, M., Ajioka, I. \& Dvorak, H. F. Vascular permeability factor/vascular endothelial growth factor (VPF/VEGF) delays and induces escape from senescence in human dermal microvascular endothelial cells. Oncogene 14, 2025-2032 (1997).

10. Simons, M., Gordon, E. \& Claesson-Welsh, L. Mechanisms and regulation of endothelial VEGF receptor signalling. Nat. Rev. Mol. Cell Biol. 17, 611 (2016).

11. Jubb, A. M. et al. Neuropilin-1 expression in cancer and development. J. Pathol. 226, 50-60 (2012).

12. Roy, S. et al. Multifaceted role of neuropilins in the immune system: potential targets for immunotherapy. Front. Immunol. 8, https://doi.org/10.3389/ fimmu.2017.01228 (2017)

13. Cébe-Suarez, S., Zehnder-Fjällman, A. \& Ballmer-Hofer, K. The role of VEGF receptors in angiogenesis; complex partnerships. Cell. Mol. Life Sci. 63, 601-615 (2006).

14. Herzog, B. et al. VEGF binding to NRP1 is essential for VEGF stimulation of endothelial cell migration, complex formation between NRP1 and VEGFR2, and signaling via FAK Tyr407 phosphorylation. Mol. Biol. Cell 22, 2766-2776 (2011).

15. Beck, B. et al. A vascular niche and a VEGF-Nrp1 loop regulate the initiation and stemness of skin tumours. Nature 478, 399-403 (2011).

16. Cao, Y. et al. VEGF exerts an angiogenesis-independent function in cancer cells to promote their malignant progression. Cancer Res. 72, 3912-3918 (2012).

17. Luo, M. et al. VEGF/NRP-1 axis promotes progression of breast cancer via enhancement of epithelial-mesenchymal transition and activation of NF-KB and B-catenin. Cancer Lett. 373, 1-11 (2016).

18. Zhang, L. et al. VEGF-A/Neuropilin 1 pathway confers cancer stemness via activating Wnt/ $\beta$-Catenin axis in breast cancer cells. Cell Physiol. Biochem. 44, 1251-1262 (2017).

19. Grun, D., Adhikary, G. \& Eckert, R. L. VEGF-A acts via neuropilin-1 to enhance epidermal cancer stem cell survival and formation of aggressive and highly vascularized tumors. Oncogene 35, 4379-4387 (2016).

20. Arbiser, J. L. et al. Overexpression of VEGF 121 in immortalized endothelial cells causes conversion to slowly growing angiosarcoma and high level expression of the VEGF receptors VEGFR-1 and VEGFR-2 in vivo. Am. J. Pathol. 156, 1469-1476 (2000).

21. Sherwood, L. M., Parris, E. E. \& Folkman, J. Tumor angiogenesis: therapeutic implications. N. Engl. J. Med. 285, 1182-1186 (1971).

22. Rosen, L. S. VEGF-targeted therapy: therapeutic potential and recent advances. Oncologist 10, 382-391 (2005).

23. Bergers, G. \& Hanahan, D. Modes of resistance to anti-angiogenic therapy. Nat. Rev. Cancer 8, 592-603 (2008). 
24. Faes, S., Santoro, T., Demartines, N. \& Dormond, O. Evolving significance and future relevance of anti-angiogenic activity of mTOR inhibitors in cancer therapy. Cancers 9, 152 (2017).

25. Guba, M. et al. Rapamycin inhibits primary and metastatic tumor growth by antiangiogenesis: involvement of vascular endothelial growth factor. Nat. Med. 8, 128 (2002).

26. Del Bufalo, D. et al. Antiangiogenic potential of the mammalian target of rapamycin inhibitor temsirolimus. Cancer Res. 66, 5549-5554 (2006).

27. Mabuchi, S. et al. RAD001 (Everolimus) delays tumor onset and progression in a transgenic mouse model of ovarian cancer. Cancer Res. 67, 2408-2413 (2007).

28. Huynh, H. et al. RAD001 (everolimus) inhibits tumour growth in xenograft models of human hepatocellular carcinoma. J. Cell. Mol. Med. 13, 1371-1380 (2009).

29. Conciatori, F. et al. Role of mTOR signaling in tumor microenvironment: an overview. Int. J. Mol. Sci. 19, 2453 (2018).

30. Iommarini, L., Porcelli, A. M., Gasparre, G. \& Kurelac, I. Non-canonical mechanisms regulating hypoxia-inducible factor 1 alpha in cancer. Front. Oncol. 7, https://doi. org/10.3389/fonc.2017.00286 (2017).

31. Frost, P. et al. Mammalian target of rapamycin inhibitors induce tumor cell Apoptosis in vivo primarily by inhibiting VEGF expression and angiogenesis. J. Oncol. 2013, 1-12 (2013).

32. Soker, S., Gollamudi-Payne, S., Fidder, H., Charmahelli, H. \& Klagsbrun, M. Inhibition of vascular endothelial growth factor (VEGF)-induced endothelial cell proliferation by a peptide corresponding to the exon 7-encoded domain of VEGF165. J. Biol. Chem. 272, 31582-31588 (1997).

33. Barr, M. P. et al. A peptide corresponding to the neuropilin-1-binding site on VEGF165 induces apoptosis of neuropilin-1-expressing breast tumour cells. Br. J. Cancer 92, 328-333 (2005).

34. Jia, H. et al. Characterization of a bicyclic peptide Neuropilin-1 (NP-1) antagonist (EG3287) reveals importance of vascular endothelial growth factor exon 8 for NP1 binding and role of NP-1 in KDR signaling. J. Biol. Chem. 281, 13493-13502 (2006).

35. Starzec, A. et al. Antiangiogenic and antitumor activities of peptide inhibiting the vascular endothelial growth factor binding to neuropilin-1. Life Sci. 79, 2370-2381 (2006).

36. Jarvis, A. et al. Small molecule inhibitors of the neuropilin-1 vascular endothelial growth factor A (VEGF-A) interaction. J. Med. Chem. 53, 2215-2226 (2010).

37. Miyauchi, J. T. et al. Ablation of Neuropilin 1 from glioma-associated microglia and macrophages slows tumor progression. Oncotarget 7, https://doi.org/ 10.18632/oncotarget.6877 (2016).

38. Powell, J. et al. Small molecule Neuropilin-1 antagonists combine antiangiogenic and antitumor activity with immune modulation through reduction of transforming growth factor beta (TGF $\beta$ ) production in regulatory T-cells. J. Med. Chem. 61, 4135-4154 (2018)

39. Nissen, J. C., Selwood, D. L. \& Tsirka, S. E. Tuftsin signals through its receptor neuropilin-1 via the transforming growth factor beta pathway. J. Neurochemistry 127, 394-402 (2013).

40. Jaafar-Maalej, C., Diab, R., Andrieu, V., Elaissari, A. \& Fessi, H. Ethanol injection method for hydrophilic and lipophilic drug-loaded liposome preparation. J. Liposome Res. 20, 228-243 (2009).

41. Blanco, E., Shen, H. \& Ferrari, M. Principles of nanoparticle design for overcoming biological barriers to drug delivery. Nat. Biotechnol. 33, 941-951 (2015).

42. Honary, S. \& Zahir, F. Effect of zeta potential on the properties of nano-drug delivery systems-a review (part 1). Trop. J. Pharm. Res. 12, https://doi.org/ 10.4314/tjpr.v12i2.19 (2013).

43. Knudsen, E. S. et al. Pancreatic cancer cell lines as patient-derived avatars: genetic characterisation and functional utility. Gut, https://doi.org/10.1136/gutjnl-2016313133 (2017).

44. Malaney, P., Nicosia, S. V. \& Dave, V. One mouse, one patient paradigm: new avatars of personalized cancer therapy. Cancer Lett. 344, 1-12 (2014).

45. Murphy, B. et al. Evaluation of alternative in vivo drug screening methodology: a single mouse analysis. Cancer Res. 76, 5798-5809 (2016).

46. Bianchi, M. et al. Distribution of metastatic sites in renal cell carcinoma: a population-based analysis. Ann. Oncol. 23, 973-980 (2012).

47. Siegel, R. L., Miller, K. D. \& Jemal, A. Cancer statistics, 2019. Cancer J. Clin. 69, 7-34 (2019).

48. Bukowski, R. M. Natural history and therapy of metastatic renal cell carcinoma: the role of interleukin-2. Cancer 80, 1198-1220 (1997)

49. Goyal, R., Gersbach, E., Yang, X. J. \& Rohan, S. M. Differential diagnosis of renal tumors with clear cytoplasm: clinical relevance of renal tumor subclassification in the era of targeted therapies and personalized medicine. Arch. Pathol. Lab. Med. 137, 467-480 (2013).
50. Woldemeskel, M. Renal cell carcinoma in humans and animals: a brief literature review. J. Clin. Exp. Pathol. 03, https://doi.org/10.4172/2161-0681.s7-001 (2013).

51. Heng, D. Y. The next 10 years: challenges for the future and overcoming resistance to targeted therapies for renal cell carcinoma. Can. Urol. Assoc. J. 10, S256-S258 (2016).

52. Gore, M. E. \& Larkin, J. M. Challenges and opportunities for converting renal cell carcinoma into a chronic disease with targeted therapies. Br. J. Cancer 104, 399-406 (2011)

53. Cho, D. C. Therapeutic challenges in advanced renal cell carcinoma. Clin. Pract. (Lond.) 10, 39-46 (2013).

54. Motzer, R. J. et al. Nivolumab versus Everolimus in advanced renal-cell carcinoma. N. Engl. J. Med. 373, 1803-1813 (2015).

55. Motzer, R. J. et al. Nivolumab plus Ipilimumab versus Sunitinib in advanced renalcell carcinoma. N. Engl. J. Med. 378, 1277-1290 (2018).

56. Motzer, R. J. et al. Avelumab plus Axitinib versus Sunitinib for advanced renal-cell carcinoma. N. Engl. J. Med. 380, 1103-1115 (2019).

57. Rini, B. I. et al. Pembrolizumab plus Axitinib versus Sunitinib for advanced renalcell carcinoma. N. Engl. J. Med. 380, 1116-1127 (2019).

58. Saxton, R. A. \& Sabatini, D. M. mTOR signaling in growth, metabolism, and disease. Cell 168, 960-976 (2017).

59. Arbiser, J. L., Bonner, M. Y. \& Gilbert, L. C. Targeting the duality of cancer. NPJ Precis. Oncol. 1, https://doi.org/10.1038/s41698-017-0026-x (2017).

60. Hanna, S. C., Heathcote, S. A. \& Kim, W. Y. mTOR pathway in renal cell carcinoma. Expert Rev. Anticancer Ther. 8, 283-292 (2008).

61. Rosa, R. et al. Angiogenic and signalling proteins correlate with sensitivity to sequential treatment in renal cell cancer. Br. J. Cancer 109, 686-693 (2013).

62. Wu, S. W., Chen, P. N., Lin, C. Y., Hsieh, Y. S. \& Chang, H. R. Everolimus suppresses invasion and migration of renal cell carcinoma by inhibiting FAK activity and reversing epithelial to mesenchymal transition in vitro and in vivo. Environ. Toxicol. 32, 1888-1898 (2017).

63. Lane, H. A. et al. mTOR inhibitor RAD001 (everolimus) has antiangiogenic/vascular properties distinct from a VEGFR tyrosine kinase inhibitor. Clin. Cancer Res. 15, 1612-1622 (2009).

64. Alshaker, H. et al. Combination of RAD001 (everolimus) and docetaxel reduces prostate and breast cancer cell VEGF production and tumour vascularisation independently of sphingosine-kinase-1. Sci. Rep. 7, 3493 (2017).

65. Buti, S., Leonetti, A., Dallatomasina, A. \& Bersanelli, M. Everolimus in the management of metastatic renal cell carcinoma: an evidence-based review of its place in therapy. Core Evid. 11, 23-36 (2016).

66. Motzer, R. J. et al. Lenvatinib, everolimus, and the combination in patients with metastatic renal cell carcinoma: a randomised, phase 2, open-label, multicentre trial. Lancet Oncol. 16, 1473-1482 (2015).

67. Huijts, C. M. et al. Immunological effects of everolimus in patients with metastatic renal cell cancer. Int. J. Immunopathol. Pharmacol. 30, 341-352 (2017).

68. O'Reilly, T. et al. Evaluation of the mTOR inhibitor, everolimus, in combination with cytotoxic antitumor agents using human tumor models in vitro and in vivo. Anticancer Drugs 22, 58-78 (2011).

69. Van Raemdonck, K., Van den Steen, P. E., Liekens, S., Van Damme, J. \& Struyf, S. CXCR3 ligands in disease and therapy. Cytokine Growth Factor Rev. 26, 311-327 (2015).

70. Liu, W. et al. Elevated expression of IFN-inducible CXCR3 ligands predicts poor prognosis in patients with non-metastatic clear-cell renal cell carcinoma. Oncotarget 7, https://doi.org/10.18632/oncotarget.7468 (2016).

71. Suyama, T. et al. Up-regulation of the interferon $\gamma$ (IFN- $\gamma$ )-inducible chemokines IFN-inducible T-cell a chemoattractant and monokine induced by IFN- $\gamma$ and of their receptor CXC receptor 3 in human renal cell carcinoma. Cancer 103, 258-267 (2005)

72. Oldham, K. A. et al. T lymphocyte recruitment into renal cell carcinoma tissue: a role for chemokine receptors CXCR3, CXCR6, CCR5, and CCR6. Eur. Urol. 61, 385-394 (2012)

73. Liotta, F. et al. Frequency of regulatory T cells in peripheral blood and in tumourinfiltrating lymphocytes correlates with poor prognosis in renal cell carcinoma. BJU Int. 107, 1500-1506 (2011).

74. Griffiths, R. W. et al. Frequency of regulatory T cells in renal cell carcinoma patients and investigation of correlation with survival. Cancer Immunol., Immunother. 56, 1743-1753 (2007).

75. Utsumi, T. et al. The association of CXCR3 and renal cell carcinoma metastasis. J. Urol. 192, 567-574 (2014).

76. Boström, A.-K., Lindgren, D., Johansson, M. E. \& Axelson, H. Effects of TGF- $\beta$ signaling in clear cell renal cell carcinoma cells. Biochem. Biophys. Res. Commun. 435, 126-133 (2013).

77. Sitaram, R. T., Mallikarjuna, P., Landström, M. \& Ljungberg, B. Transforming growth factor- $\beta$ promotes aggressiveness and invasion of clear cell renal cell carcinoma. Oncotarget 7, https://doi.org/10.18632/oncotarget.9177 (2016). 
78. Pal, K., Madamsetty, V. S., Dutta, S. K. \& Mukhopadhyay, D. Co-delivery of everolimus and vinorelbine via a tumor-targeted liposomal formulation inhibits tumor growth and metastasis in RCC. Int J. Nanomed. 14, 5109-5123 (2019).

\section{ACKNOWLEDGEMENTS}

This work was supported by $\mathrm{NIH}$ grants CA78383 and CA150190 and Florida Department of Health Cancer Research Chair Fund Florida \#3J (to D.M.). The authors would like to thank Brandy Edenfield and Laura Lewis-Tuffin for immunohistochemistry and assistance with digitization of the slides respectively.

\section{AUTHOR CONTRIBUTIONS}

K.P. designed the study, performed in vitro and in vivo experiments, acquired and interpreted the data, and wrote the manuscript. V.S.M. designed the study, developed and characterized the liposomal formulations, acquired and interpreted the data, and reviewed the manuscript. S.K.D. helped in animal experiments. R.S.A and E.W. helped in GPCR experiments. D.M. was responsible for overall designing and supervision of the work and review of the manuscript. All authors have read and approved the final manuscript.

\section{COMPETING INTERESTS}

The authors declare the following competing financial interest(s): K.P., V.S.M., and D. $M$. have applied for protection of intellectual property related to the results in the manuscript. There are no other conflicts of interest to declare.

\section{ADDITIONAL INFORMATION}

Supplementary information is available for this paper at https://doi.org/10.1038/ s41698-019-0105-2.

Correspondence and requests for materials should be addressed to D.M.

Reprints and permission information is available at http://www.nature.com/ reprints

Publisher's note Springer Nature remains neutral with regard to jurisdictional claims in published maps and institutional affiliations. Attribution 4.0 International License, which permits use, sharing, adaptation, distribution and reproduction in any medium or format, as long as you give appropriate credit to the original author(s) and the source, provide a link to the Creative Commons license, and indicate if changes were made. The images or other third party material in this article are included in the article's Creative Commons license, unless indicated otherwise in a credit line to the material. If material is not included in the article's Creative Commons license and your intended use is not permitted by statutory regulation or exceeds the permitted use, you will need to obtain permission directly from the copyright holder. To view a copy of this license, visit http://creativecommons. org/licenses/by/4.0/.

(c) The Author(s) 2019 